University of Wollongong

Research Online

Faculty of Science, Medicine and Health -

Papers: Part B

Faculty of Science, Medicine and Health

$1-1-2014$

\title{
Effects of early social isolation on the behaviour and performance of juvenile lizards, chamaeleo calyptratus
}

\author{
Cissy Ballen \\ University of Sydney, cissy.ballen@sydney.edu.au \\ Richard Shine \\ University of Sydney, rick.shine@sydney.edu.au \\ Mats M. Olsson \\ University of Sydney, molsson@uow.edu.au
}

Follow this and additional works at: https://ro.uow.edu.au/smhpapers1

\section{Publication Details Citation}

Ballen, C., Shine, R., \& Olsson, M. M. (2014). Effects of early social isolation on the behaviour and performance of juvenile lizards, chamaeleo calyptratus. Faculty of Science, Medicine and Health - Papers: Part B. Retrieved from https://ro.uow.edu.au/smhpapers1/996 


\title{
Effects of early social isolation on the behaviour and performance of juvenile lizards, chamaeleo calyptratus
}

\author{
Abstract \\ Although reptiles have traditionally been viewed as asocial, the recent discovery of complex social \\ systems in lizards suggests that an animal's social behaviour may be shaped by its interactions with \\ conspecifics early in life, as occurs in endothermic vertebrates. We reared hatchling veiled chameleons, \\ Chamaeleo calyptratus, either in isolation or in groups of four, using a split-clutch design. Social \\ interactions during the first 2 months of life substantially affected a chameleon's subsequent responses \\ to newly encountered conspecifics in standardized trials: animals reared in isolation were more \\ submissive, and adopted darker and duller colours. Isolation-reared lizards also performed less well in a \\ foraging task. Thus, social isolation early in life can impair the development of squamate reptiles, as it \\ does in mammals and birds.

\section{Publication Details} \\ Ballen, C., Shine, R. \& Olsson, M. (2014). Effects of early social isolation on the behaviour and \\ performance of juvenile lizards, chamaeleo calyptratus. Animal Behaviour, 88 1-6.
}


Effects of early social isolation on the behaviour and performance of juvenile lizards (Chamaeleo calyptratus)

Cissy J. Ballen*, Richard Shine, Mats M. Olsson

School of Biological Sciences A08, University of Sydney, NSW 2006, Australia

*E-mail: balle027@gmail.com

Manuscript for consideration as a Research Article in Animal Behaviour

10 July, 2013

Word count: 5,112 


\section{ABSTRACT}

Although reptiles traditionally have been viewed as asocial, the recent discovery of complex social systems in lizards suggests that an animal's social behaviour may be shaped by its interactions with conspecifics early in life, as occurs in endothermic vertebrates. We reared hatchling veiled chameleons (Chamaeleo calyptratus) either in isolation or in groups of four, using a split-clutch design. Social interactions during the first two months of life substantially affected a chameleon's subsequent responses to newly-encountered conspecifics in standardised trials: animals reared in isolation were more submissive, and adopted darker and duller colours. Isolation-reared lizards also performed less well in a foraging task. Thus, social isolation early in life can impair the development of squamate reptiles, as it does in mammals and birds.

Key words. - social flexibility, ontogeny of social behaviour, colour, social isolation, lizard 


\section{INTRODUCTION}

In many vertebrate species, social interactions play a central role in every individual's life, and exposure to conspecifics is critically important in allowing behavioural development during ontogeny. Thus, individuals that are reared in social isolation for the first several months of life exhibit severe impairment of social behaviour (Lukkes et al. 2009), mental performance (Einon 1980), and foraging ability (Heidbreder et al. 2000) in many species of birds and mammals (Beach 1968; Broom \& Leaver 1978; Rosen \& Hart 1963).

To our knowledge, the effects of social isolation on behavioural ontogeny have never been explored in ectothermic vertebrates, despite accumulating evidence that many reptiles exhibit complex social systems that include, for example, longterm monogamy (Bull 2000), extended parental defense of offspring (O'Connor \& Shine 2003), and individual and kin recognition (Clark 2004; Main \& Bull 1996; O'Connor \& Shine 2006). Many reptiles spend the first few months of their life with siblings or as part of a family group (Burghardt, Greene \& Rand 1977), sometimes containing many individuals (Stow et al. 2001). Juveniles often engage in intense agonistic interactions with each other (Stamps 1983a; Stamps 1983b). By analogy with "social" birds and mammals, we might expect that cognitive development in at least some reptiles thus would be affected by social interactions during early ontogeny.

As a study animal, we selected chameleons. Large clutch sizes (Andrews 2008) and relatively poor dispersal abilities (Krysko, Enge \& King 2004) suggest that hatchling chameleons often may encounter each other in the field. Although specific data are lacking for most species, hatchling chameleons are known to aggregate briefly in the wild as a result of synchronous hatching within clutches (Andrews 2004; az-Paniagua 2007; Karsten et al. 2008). Conveniently for our study, chameleons use colour change to signal dominance and submission, 
allowing us to obtain objective measures of socially-relevant signals (Schmidt 2001; Stuart-Fox \& Moussalli 2008). Additionally, the rapid growth and early maturation of most chameleon species (Schmidt 2001) means that even a relatively short-term study can encompass a high proportion of juvenile life.

\section{METHODS}

\section{Study species}

Veiled chameleons, Chamaeleo calyptratus, are large and colorful arboreal lizards that are native to western Yemen and southern Saudi Arabia (De Vosjoli \& Ferguson 1995). Despite their popularity in zoos and as pets, we know little about the behavioural ecology of this species in their native range, because fieldwork is difficult in the areas that they inhabit. In captivity, both male and female chameleons (including $C$. calyptratus) are intolerant of conspecifics and readily display to them (De Vosjoli \& Ferguson 1995). Our captive veiled chameleons exhibited aggression towards conspecifics (of both sexes) in all age groups, from hatchlings through to adults (pers. obs.).

\section{Animal husbandry}

In September 2011, we collected recently-oviposited eggs from captive chameleons at the University of Sydney and placed the eggs into either 'cold' $\left(25^{\circ} \mathrm{C}\right)$ or 'warm' $\left(28^{\circ} \mathrm{C}\right)$ incubation conditions, using a split-clutch design. The effects of incubation temperature on behaviour of the offspring are reported elsewhere (Ballen et al., in prep). Incubation temperature did not affect any of the conclusions in the current paper (i.e., there were no significant interactions between incubation temperature and rearing condition [isolation vs. group]). Our analyses included incubation temperature as an additional predictor variable, to remove any influence of this factor, but (for simplicity) we do not report these non-significant effects in the present manuscript. 
After hatching, neonates were weighed and measured (mean mass $=1.02 \pm 0.13$ g, mean SVL $=33.3 \pm 2.26 \mathrm{~mm}$ ), then randomly placed into either isolated terraria $(170 \times 190 \times 240 \mathrm{~mm})$ or in group enclosures with three other animals. The group enclosures were approximately four times the size of the isolated containers ( $200 \times 330 \times 510 \mathrm{~mm})$, to ensure that chameleons in both treatments had approximately equal space per animal. One overhead 40-W heat lamp was allocated to each group enclosure, or shared equally among a cluster of four isolated enclosures. All chameleons were fed crickets dusted with calcium and multivitamins ad libitum every day, and sprayed with a mist of water twice daily.

\section{Latency to begin foraging}

Chameleon foraging ability was tested when animals ate for the first time (age 35 days) using crickets that were $<3 \mathrm{~mm}$, and again when the animals were one month (30 days) and two months (60 days) of age, using crickets that were 3-5 $\mathrm{mm}$ in length. Foraging ability was measured as the lizard's latency of approach, or the duration of time from when the focal animal was exposed to the crickets until when it attempted capture using characteristic 'ballistic' tongue movement. At the beginning of an individual's trial, a single lizard was placed under an opaque hide in the middle of a sterile empty enclosure (170 x $190 \times 240 \mathrm{~mm})$ and allowed five minutes to settle before the hide was lifted and the lizard was exposed to three crickets moving in the container. A camera was fixed directly above the experimental enclosure to record foraging trials. We scored 769 video recordings for behavioural analysis. The trial stopped when an animal successfully captured a cricket.

\section{Social behaviour}

Two months after hatching (May 2012), we set up staged interactions between males ( $n=15$ contests between 22 individuals), and between females ( $n=11$ contests between 13 individuals). We used two animals of approximately equal body size (mass) in each contest. The maximum difference in mass ( $\mathrm{g}$ ) between 
contestants was $11.0 \%$ (mean disparity in mass $=4.9 \pm 6.4 \% \mathrm{SE}$ ). All contests were between two lizards of the same sex, and each contest included one individual reared in isolation and one individual reared in a group. The discrepancy in sample sizes between sexes was due to a skewed sex ratio at hatching, plus rapid growth rates that resulted in divergence in body sizes of animals that hatched only a short period apart. Some individuals were used in more than one contest, and so we included individual ID as a random variable to control for pseudoreplication in the analysis. At $25^{\circ} \mathrm{C}$ (the mean selected body temperature for this species (De Vosjoli \& Ferguson 1995), two individuals were placed on opposite sides of an enclosure (170 x $190 \times 240 \mathrm{~mm})$ and beneath separate hides without visual or olfactory access to one another for $15 \mathrm{~min}$ prior to each trial. After the resting period, hides were lifted and animals were able to interact.

Chameleons immediately responded to conspecifics, as evidenced by colour change and signal modifiers such as lateral flattening, rocking back and forth, gaping, and rapidly curling/uncurling tails. In the current experiment, we quantified aggression and submission during chameleon interactions using the following behaviours and associated weighted scores (numbers in parentheses show weighting):

Aggressive behaviours

1. 'Chase': the chameleon directly approaches its opponent (1).

2. 'Tail display': the chameleon quickly curls and uncurl its tail (which, when extended, is approximately the same length as its body (2)

3. 'Gape': the chameleon opens its mouth towards its opponent and rocks back and forth (2).

4. 'Lateral display': the chameleon responds to its opponent with body compressions, body tilting, and throat extensions. This behaviour is usually coupled with dramatic colour changes and forwards-backwards rocking (3).

Submissive behaviours 
1. 'Flee': the chameleon runs away from its opponent (1).

2. 'Ball curl': the chameleon lies on its side motionless, typically adopting solid black coloration (2).

A camera was fixed directly above the experimental enclosure to record social trials. Based on animals' responses within the 20-minute contest duration, we assigned each individual an aggression score and a submission score. Three colour measurements were taken consecutively at the end of the contest, while the animal was exhibiting dominance or submission. Thus, all animals had equal exposure to competitors before colour quantification. We were unable to quantify "resting" colours of the animals, because they respond to approaching observers with rapid colour change that does not resemble resting colour. In contrast, during social interactions the animals ignored the observer and thus, their colours were readily measured. No lizards were bitten or injured during these interactions. All experimental procedures were approved by the Animal Ethics Committee of the University of Sydney, Australia.

\section{Quantification of Colour}

When chameleons maintained winning or losing behaviour for at least 20 seconds at the end of the contest, we took three reflectance measurements from one location on the body using a USB2000 spectrometer (Ocean Optics Inc., Dunedin, FL, USA), with an R200 fiber optic reflectance probe and cylindrical plastic sheath to block out external light, with a PX-2 pulsed xenon lamp as a light source. Dark and reference scans from a WS-2 white standard were obtained before each set of measurements. The fiber optic probe was held at $90^{\circ}$ on a probe mount to ensure readings were taken exactly $4 \mathrm{~mm}$ from the body surface. Using 00IBase32 spec software (Ocean Optics Inc.), reflectance measurements were obtained on the fourth quadrant of an individual's torso (on the side facing the competitor: Figure 1). The diameter of each measurement was $3 \mathrm{~mm}$, and we used the average of these measurements for our analyses. We quantified green-yellow (GY) brightness and saturation from the raw reflectance 
data. Average GY brightness is the average reflectance value from R500-600. GY saturation was calculated using the formula R500-600/R300-700 (Hill \& McGraw 2006).

\section{STATISTICAL ANALYSIS}

\section{Latency to begin foraging}

In all analyses we included two factors: incubation temperature of the eggs (25 vs. $28^{\circ} \mathrm{C}$ ) and rearing condition of the hatchlings (isolated vs. group). For the traits we consider here, there were no interactions between incubation temperature and other variables (such as rearing condition or sex), so we do not report these incubation effects. Instead, we focus on the main effects of rearing conditions and sex, and their interactions. We also included mother ID and individual ID in the analyses as random factors. To explore differences in foraging behaviour between treatments, we ran mixed model analyses entering offspring ID and dams as random variables with rearing environment (isolated versus group-rearing), sex, temperature $\left(25\right.$ vs. $\left.28^{\circ} \mathrm{C}\right)$, age, and each individual's sequential trial number (1-5) as fixed factors. We also included possible interactions in the analyses. Log-transformed body mass was included as a covariate (SAS/STAT, 9.1, User's Guide, Vol. 4. 2004. SAS Institute Inc., Cary, NC. USA.).

\section{Social behaviour and colour}

To explore the determinants of variation in behaviour (log-transformed scores for aggression and submission) and colour (GY saturation, GY brightness; Fig. 1), we ran mixed model analyses entering offspring ID and dams as random variables, and sex, rearing environment (social versus isolation), temperature $\left(25\right.$ vs. $\left.28^{\circ} \mathrm{C}\right)$, and their interaction as fixed factors. Log-transformed body mass was included as a covariate. Random effects were tested for significance by removing one random factor at a time and taking the difference between the -2 log likelihoods. This was tested against a chi-square distribution with one degree 
of freedom (per removed random factor; p. 2741, SAS/STAT, 9.1, User's Guide, Vol. 4).

\section{RESULTS}

Latency to begin foraging

Latency to attack (the amount of time it took for animals to capture prey after the prey was dropped in the enclosure) was affected by rearing environment, with group-reared animals seizing their prey sooner during solitary experiments than did their isolation-reared siblings $\left(\mathrm{F}_{1,40}=4.19, \mathrm{P}=0.047\right.$; Fig. 2$)$. Latency to attack was also affected by a lizard's age, with older individuals capturing prey sooner than did younger conspecifics $\left(\mathrm{F}_{2,595}=13.50, \mathrm{P}<0.0001\right)$.

\section{Social behaviour and colour}

The level of aggression that a lizard exhibited during our standardised trials did not differ significantly between animals that had been reared in groups versus in social isolation $\left(\mathrm{F}_{1,28}=0.08, \mathrm{P}=0.786\right.$; Fig. 3$)$. However, individuals reared in isolation displayed more submissive behaviours than did individuals that had been reared with conspecifics $\left(\mathrm{F}_{1,32}=8.80, \mathrm{P}=0.006\right)$. Group-reared animals also exhibited a higher chroma (GY saturation) and were brighter (GY chroma: $F_{1,34}=$ 8.77, $\mathrm{P}=0.006$; GY brightness: $\mathrm{F}_{1,33}=14.08, \mathrm{P}=0.001$; Fig. 3 ). To summarise, lizards reared in isolation were not only more submissive, but they also adopted darker and less green colours than did conspecifics that had been reared in groups.

\section{DISCUSSION}


Recent decades have seen a dramatic paradigm shift in ideas about social complexity in "lower" vertebrates such as squamate reptiles (Leal \& Powell 2012; Wilkinson et al. 2010). Early research typically assumed that reptiles were "simple" socially, with limited behavioural flexibility (review in Northcutt \& Heath 1971). Accordingly, behavioural traits of reptiles were generally viewed as highly stereotyped fixed-action-patterns, differing among species but not changing in response to the conditions that an individual experienced during its lifetime (Glickman \& Sroges 1966). That paradigm has been replaced by an increasing appreciation of the subtlety, flexibility and complexity of reptile behaviour. Rather than slow machine-like creatures, squamate reptiles are capable of flexibly adjusting their behaviour to local conditions; and thus, the responses of reptiles are strongly influenced by local environments as well as by heritable (genetic) factors (Stamps \& Groothuis 2010).

Ontogenetic flexibility by squamates is manifested in many traits, and in response to many external factors. Unsurprisingly, traits such as growth rates and ages at maturity (and thus, of the onset of sexual behaviour) are highly sensitive to nutritional and thermoregulatory opportunities (Sinervo \& Adolph 1989). Thermal and hydric conditions during egg incubation also can exert profound effects on phenotypic traits of the resultant hatchlings (Elphick \& Shine 1998) including behaviour (Amiel \& Shine 2012; Burger 1991). Local environmental conditions also can drive major shifts in behaviours such as thermoregulatory tactics (Huey 1982). Cues from conspecifics can influence "social" behaviours such as mate-selection criteria in garter snakes Thamnophis sirtalis (Shine et al. 2006) and the specific form of visual (head-bobbing) signals in the lizard Anolis carolinenesis (Decourcy \& Jenssen 1994).

Our study reveals similar flexibility in young chameleons, as a function of their social interactions early in life. Juvenile chameleons that had been reared in isolation were less competent in their interactions with conspecifics, fleeing and curling into a ball during social trials. In contrast, their siblings that had been reared in groups interacted with each other, and displayed colours, in ways that increased their abilities to win battles against conspecifics. Thus, encounters with conspecifics early in life may shape a chameleon's subsequent behaviour, as 
it does in many species of endothermic vertebrates. Intriguingly, a young lizard's social experience also affected its performance in a foraging trial. The neonates that were reared in social groups (rather than isolation) may have benefited from early exposure to competition from conspecifics, favouring behaviours that enable a lizard to seize a prey item before it is taken by a conspecific; and familiarity with intraspecific agonistic encounters may also have facilitated an individual's performance in social tasks.

Our results challenge the implicit assumption that early social experience affects behaviour (and specifically, an animal's social competence) only in "higher" vertebrates such as mammals and birds. Instead, social tactics and abilities may be flexibly driven by early social experience in a wide range of species, including the squamate reptiles that traditionally have been viewed as too "simple" and "inflexible" to exhibit such sensitivity. To truly understand how reptiles interact with each other, we will need to abandon the view that their social behaviours are dictated largely or entirely by genes. More generally, ideas that squamates are behaviourally simple and inflexible are inconsistent with emerging evidence of their capacity to solve cognitive tasks (Amiel \& Shine 2012; Leal \& Powell 2012; Wilkinson, Chan \& Hall 2007), exhibit social learning (Wilkinson et al. 2010), and demonstrate complex intraspecific variation not only in body sizes, shapes and dietary habits (Somaweera et al. 2011; Verwaijen \& Van Damme 2008), but also in mating systems (Huang et al. 2011; Shine \& Fitzgerald 1995).

We doubt that veiled chameleons are unique in their ability to adjust their social tactics in response to local conditions. Indeed, so far as we know, chameleons exhibit simpler social systems than do many other lizards, notably viviparous scincid species that live in groups of closely-related kin (Gardner et al. 2001). In such taxa, individuals may spend much of their time in close proximity to their parents, to older relatives, and to siblings and other young conspecifics (Gardner et al. 2001; Main \& Bull 1996). In at least some of these species, individuals can recognise other individuals based upon either genetic relatedness (Clark 2004) or familiarity (Font \& Desfilis 2002). Plausibly, rearing such animals in isolation might severely impair their abilities at subsequent social interactions, as it would in the highly social mammals and birds that have attracted intensive studies in 
this respect. More generally, future research could usefully explore the possibility that squamate reptiles are far more responsive to social cues than we have expected; and hence, experimental methods that have clarified important phenomena in endotherms (such as isolation-rearing) could usefully be extended to ectothermic taxa that have been regarded as unworthy of study in such ways.

More broadly, sensitivity to early social environment may differ among species within lineages, rather than be present versus absent in entire groups. For example, a reliance upon rearing environment may be greatest for taxa that communicate using complex signals that need to be fine-tuned by external rather than genetic input (as in humans, cetaceans, some bats, and three bird orders: Beecher \& Brenowitz 2005). For example, male passerines use complex song to negotiate territory boundaries with other males. Therefore, young male passerines benefit from early exposure to conspecifics, and animals reared in isolation exhibit a reduced ability to process or produce complex social signals (Beecher 2008). Male white-crowned sparrows (Zonotrichia leucophrys nuttalli) placed in auditory isolation after hatchling, eventually (as adults) produce songs that lack fine detail, or only consist of broken whistles (Marler 1970). A similar dependence upon social interactions to fine-tune signaling may also occur in squamates that communicate via substrate vibrations (Hill 2001) or complex visual displays (Ord \& Martins 2006). In many reptiles, status recognition or previous experience overrides other traits as predictors of contest success (Burghardt 1977; Mathis \& Britzke 1999; Stuart-Fox \& Johnston 2005; Zucker \& Murray 1996). Social interactions among juveniles have rarely been documented in these species, but in others, juveniles interact frequently and establish dominance over nearby conspecifics (Stamps 1983b; Stamps 1978). The potential influence of an individual's social environment during juvenile life on its success in contests during adult life has yet to be experimentally evaluated.

What proximate mechanisms might allow the social rearing environment to generate behavioural differences in animals? In mammals, social isolation impairs cognitive development via long-term cellular and synaptic changes, which adversely affect learning, memory, and adult behavior (Baarendse et al. 
2013; Einon \& Morgan 1977; Thompson \& Heron 1954). Rearing mammals in social isolation also has profound and long-term neurochemical consequences such as abnormal dopaminergic neuronal activity and cortisol levels (Fone \& Porkess 2008). Knowing whether or not the same physiological and molecular mechanisms are involved in the effects of social isolation on reptiles - for example, their physiological responses to stress - might clarify broader issues concerning the proximate mechanisms underlying the evolutionary origins of social behavior.

Our results also have obvious implications for captive rearing of reptiles. These animals commonly are raised (by zoos, private keepers, etc.) in social isolation, under the assumption that social cues are irrelevant to ontogenetic development in these animals. Our data challenge that assumption, even for a putatively asocial lizard species. Both for animal welfare reasons and to facilitate subsequent effective breeding, we should closely examine the potential consequences of social isolation for young reptiles. More broadly, our study suggests a surprising commonality among a broad spectrum of species: an environment rich in social interaction may provide important (and as yet, poorly understood) benefits for the wellbeing of the animal.

Acknowledgements. - We thank Melanie Elphick and Matthew Greenlees for their help and support. This study was funded by the Australian Research Council [FL 120100074]. All experimental procedures were approved by University of Sydney Animal Ethics Committee (L04/4-2012/3/5678). 


\section{REFERENCES}

Amiel, J. J. \& Shine, R. 2012. Hotter nests produce smarter young lizards. Biology Letters, 8, 372-374.

Andrews, R. M. 2004. Effects of temperature and moisture on embryonic diapause of the veiled chameleon (Chamaeleo calyptratus). The Journal of experimental zoology, 301A, 629-635.

Andrews, R. M. 2008. Effects of incubation temperature on growth and performance of the veiled chameleon (Chamaeleo calyptratus). Journal of Experimental Zoology Part A: Ecological Genetics and Physiology, 309A, 435446.

az-Paniagua, C. 2007. Effect of cold temperature on the length of incubation of Chamaeleo chamaeleon. Amphibia-Reptilia, 28, 387-392.

Baarendse, P. J. J., Counotte, D. S., Donnell, P. \& Vanderschuren, L. J. M. J. 2013. Early social experience is critical for the development of cognitive control and dopamine modulation of prefrontal cortex function. Neuropsychoparmacology, 38, 1485-1494.

Beach, F. A. 1968. Coital Behavior in Dogs. III. Effects of Early Isolation on Mating in Males. Behaviour, 30, 218-238.

Beecher, M. D. 2008. Function and Mechanisms of Song Learning in Song Sparrows. In: Advances in the Study of Behavior (Ed. by H. J. Brockmann, T. Roper, M. Naguib, K. E. Wynne-Edwards, C. Barnard \& J. Mitani), pp. 167-225: Academic Press.

Beecher, M. D. \& Brenowitz, E. A. 2005. Functional aspects of song learning in songbirds. Trends in Ecology \& Evolution, 20, 143-149.

Broom, D. M. \& Leaver, J. D. 1978. Effects of group-rearing or partial isolation on later social behaviour of calves. Animal Behaviour, 26 (4), 1255-1263.

Bull, C. M. 2000. Monogamy in lizards. Behavioural Processes, 51, 7-20.

Burger, J. 1991. Effects of Incubation Temperature on Behavior of Hatchling Pine Snakes: Implications for Reptilian Distribution. Behavioral Ecology and Sociobiology, 28, 297-303.

Burghardt, G. M. 1977. Of iguanas and dinosaurs: Social behavior and communication in neonate reptiles. American Zoologist, 17, 177-190.

Burghardt, G. M., Greene, H. W. \& Rand, A. S. 1977. Social Behavior in Hatchling Green Iguanas: Life at a Reptile Rookery. Science, 195, 689-691.

Clark, R. W. 2004. Kin recognition in rattlesnakes. Proceedings of the Royal Society of London. Series B: Biological Sciences, 271, S243-S245.

De Vosjoli, P. \& Ferguson, G. 1995. Care and breeding of panther, jackson's, veiled, and parson's chameleons. Santee, California U.S.A.: Andvanced Vivarium Systems, Inc. 
Decourcy, K. R. \& Jenssen, T. A. 1994. Structure and use of male territorial headbob signals by the lizard Anolis carolinensis. Animal Behaviour, 47, 251262.

Einon, D. 1980. Spatial memory and response strategies in rats: age, sex and rearing differences in performance. Quarterly Journal of Experimental Psychology, 32, 473-489.

Einon, D. F. \& Morgan, M. J. 1977. A critical period for social isolation in the rat. Developmental Psychobiology, 10, 123-132.

Elphick, M. J. \& Shine, R. 1998. Longterm effects of incubation temperatures on the morphology and locomotor performance of hatchling lizards (Bassiana duperreyi, Scincidae). Biological Journal of the Linnean Society, 63, 429-447.

Fone, K. C. F. \& Porkess, M. V. 2008. Behavioural and neurochemical effects of post-weaning social isolation in rodents-Relevance to developmental neuropsychiatric disorders. Neuroscience \& Biobehavioral Reviews, 32, 1087 1102.

Font, E. \& Desfilis, E. 2002. Chemosensory recognition of familiar and unfamiliar conspecifics by juveniles of the Iberian wall lizard Podarcis hispanica. Ethology, 108, 319-330.

Gardner, M. G., Bull, C. M., Cooper, S. J. B. \& Duffield, G. A. 2001. Genetic evidence for a family structure in stable social aggregations of the Australian lizard Egernia stokesii. Molecular Ecology, 10, 175-183.

Glickman, S. E. \& Sroges, R. W. 1966. Curiosity in Zoo Animals. Behaviour, 26, 151-187.

Heidbreder, C. A., Weiss, I. C., Domeney, A. M., Pryce, C., Homberg, J., Hedou, G., Feldon, J., Moran, M. C. \& Nelson, P. 2000. Behavioral, neurochemical and endocrinological characterization of the early social isolation syndrome. Neuroscience, 100, 749-768.

Hill, G. E. \& McGraw, K. J. 2006. Bird Coloration: Mechanisms and measurements: Harvard University Press.

Hill, P. S. M. 2001. Vibration and animal communication: a review. American Zoologist, 41, 1135-1142.

Huang, W. S., Greene, H. W., Chang, T. J. \& Shine, R. 2011. Territorial behavior in Taiwanese kukrisnakes (Oligodon formosanus). Proceedings of the National Academy of Sciences of the United States of America. 108, 7455-7459.

Huey, R. B. 1982. Temperature, physiology and ecology of reptiles. In: Biology of the Reptilia Vol. 12 (Ed. By C. Gans \& F. H. Pough), pp. 25-91. London: Academic Press.

Karsten, K. B., Andriamandimbiarisoa, L. N., Fox, S. F. \& Raxworthy, C. J. 2008. A unique life history among tetrapods: An annual chameleon living mostly as an egg. Proceedings of the National Academy of Sciences, 105, 89808984.

Krysko, K. L., Enge, K. M. \& King, F. W. 2004. The veiled chameleon, Chamaeleo calyptratus: A new exotic lizard species in Florida. Florida Scientist, 67, 249253.

Leal, M. \& Powell, B. J. 2012. Behavioural flexibility and problem-solving in a tropical lizard. Biology Letters, 8, 28-30.

Lukkes, J. L., Mokin, M. V., Scholl, J. L. \& Forster, G. L. 2009. Adult rats exposed to early-life social isolation exhibit increased anxiety and conditioned fear 
behavior, and altered hormonal stress responses. Hormones and Behavior, 55, 248-256.

Main, A. R. \& Bull, M. C. 1996. Mother-offspring recognition in two Australian lizards,Tiliqua rugosaandEgernia stokesii. Animal Behaviour, 52, 193-200.

Marler, P. 1970. A comparative approach to vocal learning: Song development in white-crowned sparrows. Journal of Comparative and Physiological Psychology, 71, 1-25.

Mathis, A. \& Britzke, E. 1999. The roles of body size and experience in agonistic displays of the Ozark zigzag salamander, Plethodon angusticlavius. Herpetologica, 55, 344-352.

Northcutt, R. G. \& Heath, J. E. 1971. Performance of Caimans in a T-Maze. Copeia, 1971, 557-560.

O'Connor, D. \& Shine, R. 2003. Lizards in 'nuclear families': a novel reptilian social system in Egernia saxatilis (Scincidae). Molecular Ecology, 12, 743-752.

O'Connor, D. E. \& Shine, R. 2006. Kin discrimination in the social lizard Egernia saxatilis (Scincidae). Behavioral Ecology, 17, 206-211.

Ord, T. J. \& Martins, E. P. 2006. Tracing the origins of signal diversity in anole lizards: phylogenetic approaches to inferring the evolution of complex behaviour. Animal Behaviour, 71, 1411-1429.

Rosen, J. \& Hart, F. M. 1963. Effects of early social isolation upon adult timidity and dominance in Peromyscus. Psychological Reports, 13, 47-50.

Schmidt, W. 2001. Chamaeleo calyptratus: The Yemen chameleon. Münster, Germany: Matthias Schmidt Publications.

Shine, R. \& Fitzgerald, M. 1995. Variation in mating systems and sexual size dimorphism between populations of the Australian python Morelia spilota (Serpentes: Pythonidae). Oecologia, 103, 490-498.

Shine, R., Webb, J. K., Lane, A. \& Mason, R. T. 2006. Flexible mate choice: a male snake's preference for larger females is modified by the sizes of females encountered. Animal Behaviour, 71, 203-209.

Sinervo, B. \& Adolph, S. 1989. Thermal sensitivity of growth rate in hatchling Sceloporus lizards: environmental, behavioral and genetic aspects. Oecologia, 78, 411-419.

Somaweera, R., Webb, J. K., Brown, G. P. \& Shine, R. P. 2011. Hatchling Australian freshwater crocodiles rapidly learn to avoid toxic invasive cane toads. Behaviour, 148, 501-517.

Stamps, J. 1983a. The relationship between ontogenetic habitat shifts, competition and predator avoidance in a juvenile lizard (Anolis aeneus). Behavioral Ecology and Sociobiology, 12, 19-33.

Stamps, J. 1983b. Territoriality and the defence of predator-refuges in juvenile lizards. Animal Behaviour, 31, 857-870.

Stamps, J. \& Groothuis, T. G. G. 2010. The development of animal personality: relevance, concepts and perspectives. Biological Reviews, 85, 301-325.

Stamps, J. A. 1978. A field study of the ontogeny of social behavior in the lizard Anolis aeneus. Behaviour, 66, 1-31.

Stow, A. J., Sunnucks, P., Briscoe, D. A. \& Gardner, M. G. 2001. The impact of habitat fragmentation on dispersal of Cunningham's skink (Egernia cunninghami): evidence from allelic and genotypic analyses of microsatellites. Molecular Ecology, 10, 867-878. 
Stuart-Fox, D. \& Moussalli, A. 2008. Selection for social signalling drives the evolution of chameleon colour change. PLoS Biology, 6, e25.

Stuart-Fox, D. M. \& Johnston, G. R. 2005. Experience overrides colour in lizard contests. Behaviour, 142, 329-350.

Thompson, W. R. \& Heron, W. 1954. The effects of restricting early experience on the problem-solving capacity of dogs. Canadian Journal of Psychology/Revue canadienne de psychologie, 8, 17-31.

Verwaijen, D. \& Van Damme, R. 2008. Foraging mode and its flexibility in Lacertid lizards from Europe. Journal of Herpetology, 42, 124-133.

Wilkinson, A., Chan, H. M. \& Hall, G. 2007. Spatial learning and memory in the tortoise (Geochelone carbonaria). Journal of Comparative Psychology, 121, 412-418.

Wilkinson, A., Kuenstner, K., Mueller, J. \& Huber, L. 2010. Social learning in a non-social reptile (Geochelone carbonaria). Biology Letters, 6, 614-616.

Zucker, N. \& Murray, L. 1996. Determinants of dominance in the tree lizard Urosaurus ornatus: the relative importance of mass, previous experience and coloration. Ethology, 102, 812-825. 
Figure 1. In veiled chameleons, the same individual can display different colours in response to social stimuli. The arrow points to the location where colour measurements were taken with a spectrometer during social experiments in the current study.

Figure 2. Foraging latency (the amount of time required before the animal captured a prey item), for hatchling veiled chameleons over the first two months of life. Chameleons that were reared in isolation (filled circles) were slower at this foraging task than were those reared in groups (empty circles). Variation in latency was low at hatching (age 0), but diverged after the young lizards had been exposed to the two social rearing treatments after hatching.

Figure 3. Effects of sex and rearing conditions (social versus isolated) on a hatchling chameleon's response to social stimuli at two months of age.

Lizards that were isolated immediately after hatching exhibited more submissive behaviours than did group-reared animals $(\mathrm{a}, \mathrm{b})$. Isolated females were more aggressive than were females from groups (c), and group-reared males were slightly more aggressive than were isolated males (d). Isolated individuals of both sexes were less chromatic $(e, f)$ and less bright $(g, h)$ in response to social interaction. 